\title{
ELECTRON CLOUD GENERATION AND TRAPPING IN A QUADRUPOLE MAGNET AT THE LOS ALAMOS PSR*
}

\author{
R. J. Macek", A. A. Browman, and J. E. Ledford, TechSource, Inc., Santa Fe, NM, 87505, USA, \\ and Los Alamos National Laboratory, Los Alamos, NM, 87545, USA \\ M. J. Borden, J. F. O’Hara, R. C. McCrady, L. J. Rybarcyk, T. Spickermann, and T. J. Zaugg, \\ Los Alamos National Laboratory, Los Alamos, NM, 87545, USA \\ M. T. F. Pivi, Stanford Linear Accelerator Center, Menlo Park, CA, 94025, USA.
}

\section{Abstract}

A diagnostic to measure electron cloud formation and trapping in a quadrupole magnet has been developed, installed, and successfully tested at PSR. Beam studies with this diagnostic show that the electron flux striking the wall in the quadrupole is comparable to or larger than in an adjacent drift. In addition, the trapped electron signal, obtained using the sweeping feature of diagnostic, was larger than expected and decayed very slowly with an exponential time constant of 50 to $100 \mu \mathrm{s}$. Experimental results were also obtained which suggest that a significant fraction of the electrons observed in the adjacent drift space were seeded by electrons ejected from the quadrupole.

\section{INTRODUCTION}

Available evidence on the fast, transverse instability observed at the Los Alamos Proton Storage Ring (PSR) supports the hypothesis of a two-stream instability driven by coupled motion of the proton beam with low-energy clouds of electrons, hence the designation "e-p" instability $[1,2]$. In this picture, electron clouds (EC) responsible for the instability are generated by primary electrons born at the wall from beam losses (particularly grazing angle losses) that are in turn amplified by beam-induced multipactor on the trailing edge of the long proton bunch ( $\sim 270 \mathrm{~ns}$ ). However, the locations (drifts, dipoles or quadrupole magnets) of the dominant electron sources driving the instability are not fully resolved in part because of large uncertainties in the distribution of primary electrons from beam losses. An improved understanding of the EC sources would be beneficial for the development of cures by suppression of EC formation.

There are several reasons that quadrupoles may be a strong source of EC that drive the instability in PSR. 1) The number of primary electrons produced per lost proton is a strong function of $\theta$, the angle of incidence with respect to the normal to the surface $(\propto 1 / \cos \theta)$. 2) Beam loss simulations with ORBIT show that grazing angle losses will be highest in the quadrupoles where the beta functions have a maximum. 3) Electrons can be trapped in the magnetic fields of the quadrupole. 4) Simulations

\footnotetext{
* Work supported by DOE SBIR Grant No. DE-FG02-04ER84105 and CRADA No. LA05C10535 between TechSource, Inc. and the Los Alamos National Laboratory. LANL is supported by the US Department of Energy under Contract Number DE-AC52-06NA25396. \#macek@lanl.gov
}

of EC development showed that numerous electrons are ejected from the quadrupole by ExB drifts [3].

\section{QUADRUPOLE DIAGNOSTIC}

We have developed a diagnostic to measure the electron flux striking the wall during beam-induced multipactor (the prompt electron signal), and by pulsing a sweeping electrode can also measure electrons trapped in the quadrupole (swept electron signal) after the beam pulse has left the magnet. A schematic cross sectional diagram of the diagnostic is shown in Figure 1. Mechanical design and construction are described in reference [4].

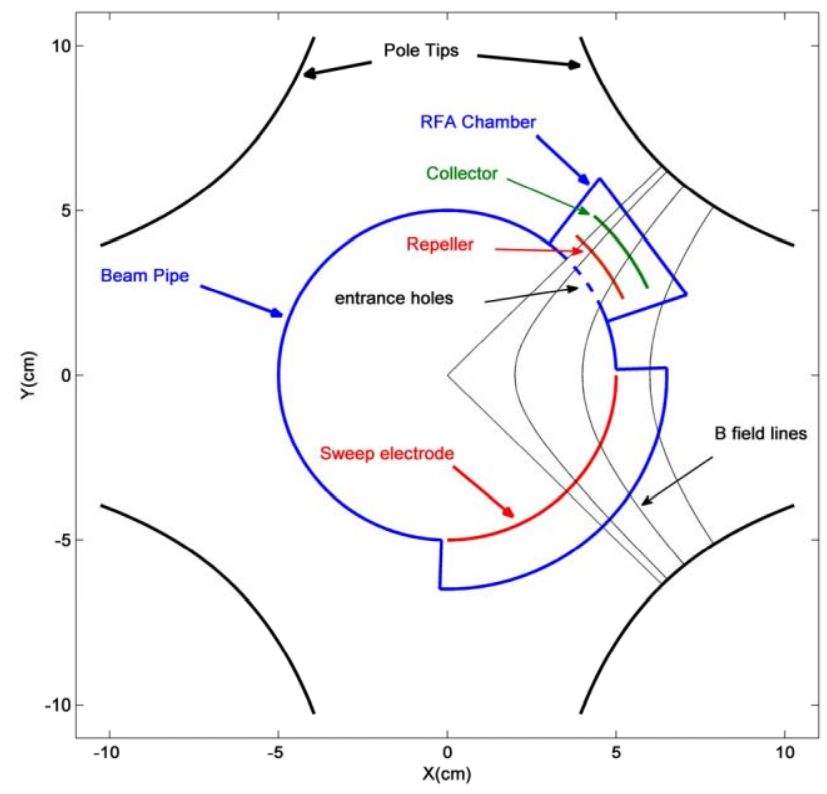

Figure 1. Schematic cross section of the electron sweeping diagnostic in a quadrupole magnet. Its principal components are a retarding field analyzer (RFA) chamber containing a repeller grid and collector plate, holes in the beam pipe at the entrance to the RFA chamber, and a high voltage sweeping electrode.

The diagnostic is an adaptation of the electron sweeping detector which we developed earlier for use in drift spaces. Electrons from the cloud execute gyro motion (typical radius $<1 \mathrm{~mm}$ ) around magnetic (B) field lines in the quadrupole and can enter the RFA chamber through holes in the beam pipe. They will get past the repeller grid if they have sufficient velocity along the magnetic field lines to overcome the negative bias on the repeller 
grid and will be collected at the collector plate to produce the detector signal. The collector plate is biased to $+45 \mathrm{~V}$ to suppress secondary at the collector. Wide bandwidth ( $1 \mathrm{kHz}$ to $50 \mathrm{MHz}$ ) front-end electronics are placed close to the magnet in the beam tunnel to amplify the collector signal for observation outside of the beam tunnel.

Electrons trapped or remaining in the quadrupole field can be observed by pulsing the sweeping electrode with a fast rise time negative voltage pulse (typically $-485 \mathrm{~V}$ ) when the beam is not present in the magnet (during the beam-free gap passage or after extraction). The signal produced by pulsing the sweeping electrode is designated the "swept electron" signal to distinguish it from the "prompt signal" (generated during the multipactor process) obtained when the sweeping electrode is grounded.

Previous experience with fast amplifiers on the collector signal showed it was necessary to give careful attention to reducing electromagnetic pickup from the beam. The entrance holes to the RFA chamber were small $(2.8 \mathrm{~mm})$ with a spacing between them sufficient to have the holes represent $\sim 20 \%$ of the surface area at the entrance, thereby leaving most of the metal intact to carry to wall currents with little additional impedance. In addition, the entrance plate was covered with 40 mesh copper screen sandwiched between the entrance plate and a copper plate with somewhat larger holes aligned with the holes in the entrance plate. The repeller grid screen was soldered to 12 ceramic chip rf capacitors which gave a good rf (AC) ground to the repeller grid. Even with these measures significant beam pickup was observed. Fortunately, the beam pickup was sufficiently reproducible from pulse to pulse to allow a background subtraction to improve the signal to noise ratio.

\section{EXPERIMENTAL RESULTS}

The electron cloud diagnostic worked well and provides good quality signals using background subtractions (obtained with repeller at $-511 \mathrm{~V}$ ) and averaging over 16 to 64 macropulses (i.e., accumulation cycles in the ring). Using this device, we were able to measure the prompt and swept (trapped) electron signals and thereby better characterize the generation and trapping of electron clouds in a quadrupole magnet. Measurements were made of the effect on the electron cloud signals of changing various beam and accelerator parameters but only a select subset of the results will be covered here.

\section{Electron signals from the diagnostic}

An example of prompt and swept signals for a production beam of $86 \mu \mathrm{A}(4.29 \mu \mathrm{C} /$ pulse at $20 \mathrm{~Hz})$ is shown in Figure 2 and compared to the proton beam current measured by a wall current monitor (designated WC41). Electrons from trailing edge multipactor give a prompt signal that peaks at the end of each proton beam pulse passage. The swept signal was produced by applying a short ( 100 ns) $-485 \mathrm{~V}$ pulse to the sweeping electrode $\sim 3.5 \mu$ s after the beam was extracted. When the prompt signal is converted to an electron flux striking the wall we find the important result that the electron flux striking the walls in the quadrupole is $\sim 1$ to 3 times larger than the flux measured in an adjacent drift space. Since simulations indicate that the beam-induced multipactor gain for electrons is about a factor of 25 or so lower in a quadrupole than in a drift space, we have evidence that the seed electrons generated by beam losses are at least a factor of 25 higher in the quadrupole than in the adjacent drift space.

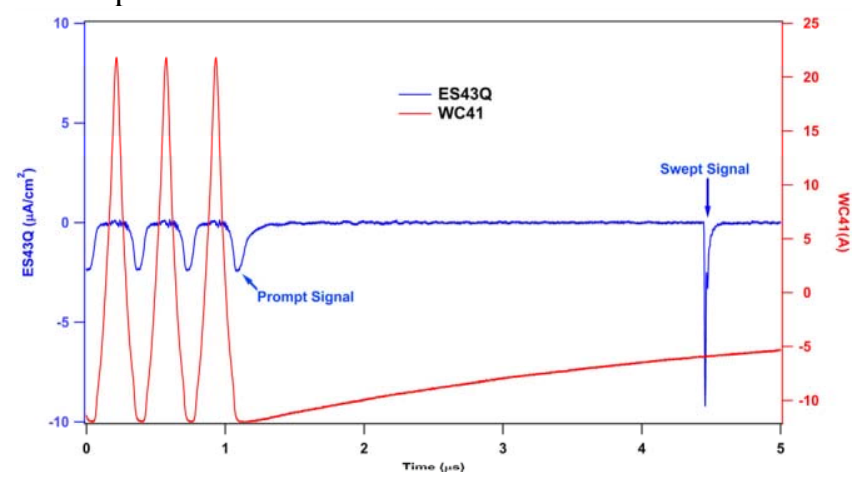

Figure 2. Prompt and swept electron signals from the quadrupole detector (designated as ES43Q) compared with the proton beam current measured with the wall current monitor (WC41). For this graph the electron detector signal has been converted to an electron flux $\left(\mu \mathrm{A} / \mathrm{cm}^{2}\right)$ striking the wall.

\section{Electrons trapped in the quadrupole}

A key motivation for the development of this diagnostic was to measure the line density and lifetime of electrons trapped in the quadrupole after the beam is extracted. Plots of the trapped (swept) electron signal amplitude as a function of time after extraction are presented in Figure 3 and show that the trapped electrons persist for a long time (200-300 $\mu$ s) with an exponential decay time of $\sim 60$ to $100 \mu \mathrm{s}$.

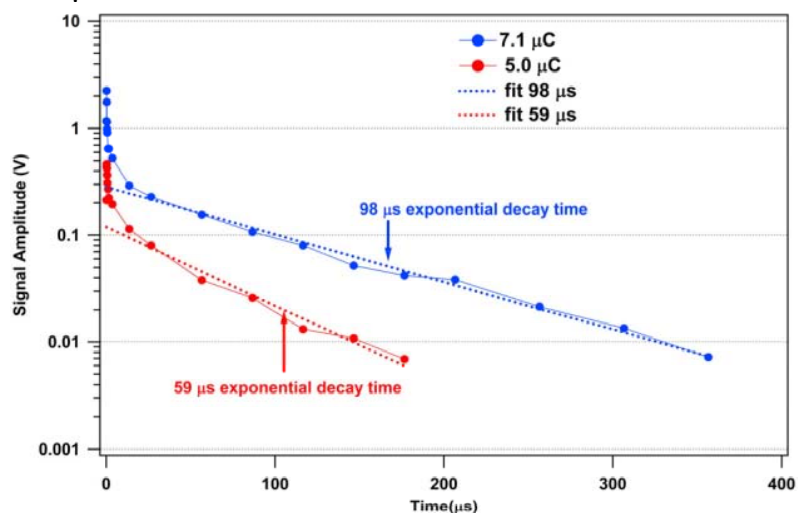

Figure 3. Trapped electron dissipation/decay curves for ES43Q amplitude data of taken at two beam intensities, $7.1 \mu \mathrm{C}$ /pulse and $5.0 \mu \mathrm{C}$ /pulse. The exponential decay times shown were obtained from fits to the portion of the curves after $\sim 15 \mu \mathrm{s}$.

The measured decay time is in reasonable agreement with simulations but the measured ratio of the swept 
signal amplitude (at $\sim 5 \mu$ after extraction) to the last prompt signal is considerably larger (factor of 5-10) than obtained in simulations. The integral of the swept electron signal obtained near the end of the $\sim 80$ ns beamfree gap between bunch passages translates into a line density of $\sim 0.6 \mathrm{nC} / \mathrm{m}$ compared with an average proton line density of $\sim 95 \mathrm{nC} / \mathrm{m}$ for the $7.1 \mu \mathrm{C}$ beam pulse.

\section{Signal variation with beam intensity}

Both swept and prompt electrons signals were measured as functions of beam intensity. Beam intensity was varied by chopping 1 out of $n$ turns of injection into the ring while all other beam and accelerator parameters were kept fixed. Plots of the data for the prompt and swept signal amplitudes as a function of beam intensity are shown in Figure 4 along with power law fits to the data. Both signal amplitudes vary strongly with intensity; the fitting gave exponents of 5.2 and 3.6 for the prompt and swept signal curves respectively. Simulations of the prompt signals at two intensities ( 5 and $7 \mu \mathrm{C} /$ pulse) for a peak SEY of 1.5 and constant fractional beam loss rate show much less variation with intensity. See reference [5] for a discussion of the comparable variation of EC signals with intensity in a drift space.

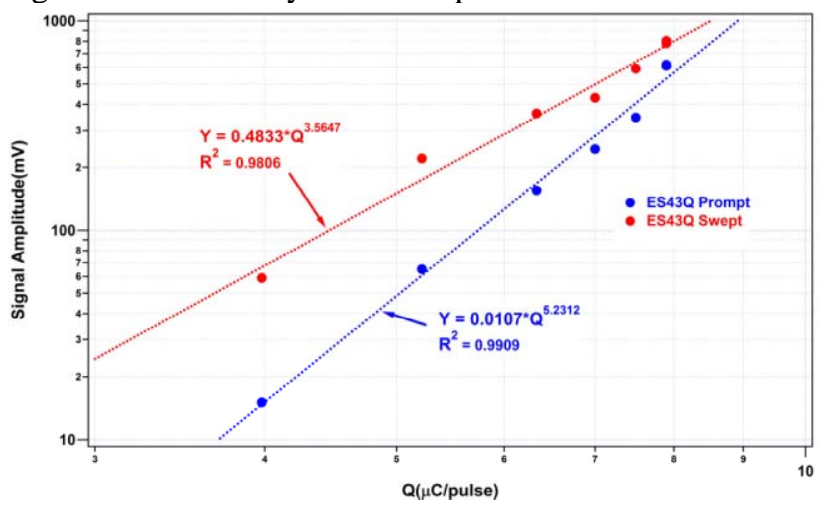

Figure 4. Variation with beam intensity of prompt and swept signals from the quadrupole diagnostic (ES43Q).

\section{Evidence for electrons ejected from quadrupole}

Another electron cloud diagnostic (labeled ES41Y RFA) located in the drift space just upstream of the quadrupole was used in conjunction with the quadrupole diagnostic (ES43Q) to obtain evidence for electrons ejected from the quadrupole. A significant reduction $(\sim 25 \%)$ of the prompt electron signal (ES41Y) in the nearby drift space was observed when the sweeping electrode in the quadrupole was pulsed at $-485 \mathrm{~V}$ for $2-3$ $\mu$ s while beam was still present in the ring as shown in Figure 5. Such a reduction would be expected if a significant fraction of the drift space signal is seeded by electrons ejected from the quadrupole rather than directly by beam losses in the drift space near the electron diagnostic. Pulsing of the sweeping electrode in the quadrupole will suppress some electron generation during multipacting and will also clear out electrons trapped in the quadrupole in the region near the electrode. This will then reduce the electrons ejected from the quadrupole by the ExB drift mechanism while the electrode is at $-485 \mathrm{~V}$.

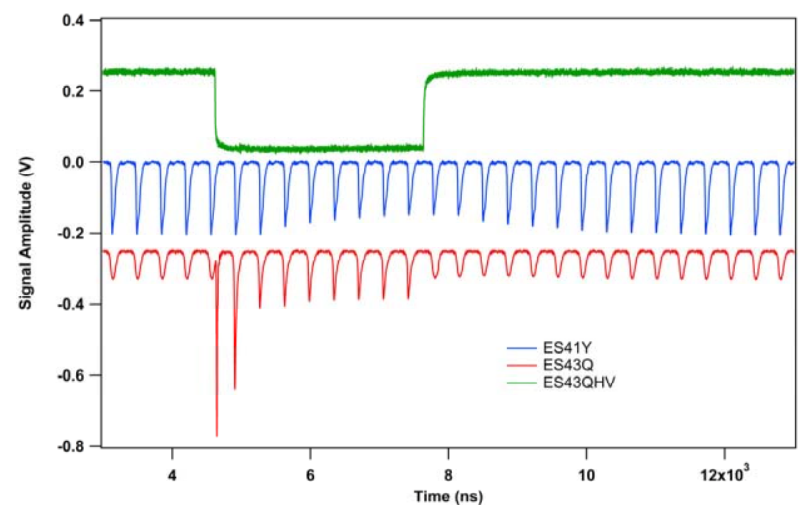

Figure 5. Evidence for seeding of the drift space region by electrons ejected from the quadrupole. The green curve (ES43QHV) is an attenuated signal from the $-485 \mathrm{~V}$ pulse applied to the sweeping electrode in the quadrupole. The corresponding collector signal from the quadrupole is the red curve (ES43Q). The blue trace is the prompt signal from the ES41Y detector in the adjacent drift space. Note the $\sim 25 \%$ reduction in the ES41Y signal after about 8 turns with the sweeping electrode energized followed by recovery after the electrode is de-energized.

\section{SUMMARY AND CONCLUSIONS}

We have developed and successfully tested a new electron cloud diagnostic which can measure both the electron flux striking the vacuum chamber wall and electrons trapped in a quadrupole field. Using this device we have found the following important results for PSR:

- The prompt electron fluxes striking the wall in a quadrupole are comparable to or larger than those in an adjacent drift space. Since the multipactor gain is estimated to be about a factor of 25 lower, the primary electrons generated in the quadrupole are thus a factor of $>25$ higher than in the drift space.

- A significant fraction of the electron cloud is trapped in the quadrupole and will survive for a long time after the beam is extracted (decay time $\sim 100 \mu \mathrm{s}$ ).

- Evidence that a sizeable fraction ( $>25 \%)$ of the electron cloud in the adjacent drift space is seeded by electrons ejected from the quadrupole.

\section{REFERENCES}

[1] D. Neuffer et al, NIM A321, p. 1(1992).

[2] R. Macek et al, "Status of Experimental Studies of Electron cloud Effects at the Los Alamos Proton Storage Ring", Proceedings of the ECLOUD'04 Workshop, p. 63-75, CERN 2005-001.

[3] R. Macek and M. T. F. Pivi, "ExB Drift of the e-cloud in a PSR Quadrupole and Dipole”, LANL Report, LA-UR-06-0283 (2005).

[4] J. F. O'Hara et al, FRPMS054, PAC'07 (2007).

[5] Y. Sato et al, THPAS013, PAC’07 (2007). 